\title{
Postmortem computed tomography and magnetic resonance imaging of an abdominal gunshot wound
}

\author{
Gascho, Dominic ; Bolliger, Stephan A ; Thali, Michael J ; Tappero, Carlo
}

\begin{abstract}
The use of postmortem computed tomography (CT) has been described in many articles concerning gunshot injuries. Postmortem magnetic resonance imaging (MRI) for better assessment of soft tissue injuries has also been mentioned in the literature, albeit much less often. The use of postmortem MRI for abdominal gunshot wounds has not been previously presented in the literature. The present case report describes the findings of an abdominal gunshot wound detected by postmortem CT and MRI, followed by an autopsy. The main imaging findings on CT were a hyperdense ring at the entrance wound, which indicated the muzzle imprint mark, a hyperdense region beneath the skin, which was suggestive of combustion residue, gas cavities surrounding the bullet path, which might be related to the temporary cavity, and a fracture of the 13th rib on the left. Magnetic resonance imaging provided a clear depiction of defects in the muscle tissue and peritoneal fat, as well as an injury to the left kidney and a large volume of blood in the abdominal cavity. Computed tomography combined with MRI provided a descriptive presentation of the intracorporeal trajectory noninvasively. Autopsy confirmed the radiologic findings but additionally revealed further relevant findings, which were not detected radiologically, such as a duodenal perforation. Autopsy also detected subendocardial hemorrhages and shock kidney, which were consistent with severe blood loss. The imaging findings and their interpretations are discussed in this case report, as well as the role of CT and MRI in the assessment of abdominal gunshot wounds compared with autopsy.
\end{abstract}

DOI: https://doi.org/10.1097/paf.0000000000000547

Posted at the Zurich Open Repository and Archive, University of Zurich

ZORA URL: https://doi.org/10.5167/uzh-188502

Journal Article

Published Version

Originally published at:

Gascho, Dominic; Bolliger, Stephan A; Thali, Michael J; Tappero, Carlo (2020). Postmortem computed tomography and magnetic resonance imaging of an abdominal gunshot wound. American Journal of Forensic Medicine and Pathology, 41(2):119-123.

DOI: https://doi.org/10.1097/paf.0000000000000547 


\title{
Postmortem Computed Tomography and Magnetic Resonance Imaging of an Abdominal Gunshot Wound
}

\author{
Dominic Gascho, RT, * Stephan A. Bolliger, MD, * Michael J. Thali, MD, * and Carlo Tappero, MD*†
}

\begin{abstract}
The use of postmortem computed tomography (CT) has been described in many articles concerning gunshot injuries. Postmortem magnetic resonance imaging (MRI) for better assessment of soft tissue injuries has also been mentioned in the literature, albeit much less often. The use of postmortem MRI for abdominal gunshot wounds has not been previously presented in the literature. The present case report describes the findings of an abdominal gunshot wound detected by postmortem CT and MRI, followed by an autopsy. The main imaging findings on CT were a hyperdense ring at the entrance wound, which indicated the muzzle imprint mark, a hyperdense region beneath the skin, which was suggestive of combustion residue, gas cavities surrounding the bullet path, which might be related to the temporary cavity, and a fracture of the 13th rib on the left. Magnetic resonance imaging provided a clear depiction of defects in the muscle tissue and peritoneal fat, as well as an injury to the left kidney and a large volume of blood in the abdominal cavity. Computed tomography combined with MRI provided a descriptive presentation of the intracorporeal trajectory noninvasively. Autopsy confirmed the radiologic findings but additionally revealed further relevant findings, which were not detected radiologically, such as a duodenal perforation. Autopsy also detected subendocardial hemorrhages and shock kidney, which were consistent with severe blood loss.

The imaging findings and their interpretations are discussed in this case report, as well as the role of CT and MRI in the assessment of abdominal gunshot wounds compared with autopsy.
\end{abstract}

Key Words: bullet, gunshot, radiologic wound ballistics, postmortem computed tomography,

postmortem magnetic resonance imaging, virtopsy

(Am J Forensic Med Pathol 2020;41: 119-123)

P ostmortem computed tomography (CT) is frequently presented as a supplementary examination to autopsy in cases of gunshot wounds. ${ }^{1,2}$ The major strength of CT is the rapid detection and location of lodged projectiles or bullet fragments inside a cadaver. $^{3-5}$ Computed tomography also allows for an in situ assessment of the bullet trajectory via the detection of osseous injuries. $^{3-5}$ For a detailed assessment of soft tissue, however, the additional use of postmortem magnetic resonance imaging (MRI) is recommended, ${ }^{3,6,7}$ although postmortem MRI has only been used in a small number of gunshot wound cases so far. ${ }^{2}$ Furthermore, the value of both imaging modalities has primarily been presented in cases of gunshot wounds to the head, whereas

Manuscript received November 25, 2019; accepted February 28, 2020. From the *Department of Forensic Medicine and Imaging, Institute of Forensic Medicine, University of Zurich, Zurich; and †Department of Radiology, Hôpital Fribourgeois, Fribourg, Switzerland.

The authors report no conflict of interest.

Reprints: Dominic Gascho, Department of Forensic Medicine and Imaging, Zurich Institute of Forensic Medicine, University of Zurich Winterthurerstrasse 190/52, CH-8057 Zurich, Switzerland. E-mail: dominic.gascho@irm.uzh.ch.

Copyright (C) 2020 Wolters Kluwer Health, Inc. All rights reserved.

ISSN: 0195-7910/20/4102-0119

DOI: 10.1097/PAF.0000000000000547 postmortem CT, and especially postmortem MRI, have rarely been described in cases of gunshot wounds to the torso. To the best of our knowledge, the use of postmortem MRI for the detection of an abdominal gunshot injury has not been previously described in the literature.

Here, we report the case of a decedent with an abdominal gunshot wound who underwent CT and MRI before autopsy. This case report describes the imaging and autopsy findings. Conclusions related to the shot distance, intracorporeal deflection of the bullet, and extent of the temporary cavity were drawn from the imaging findings. Furthermore, the additional value of MRI over CT is discussed, and the limitations of both imaging modalities compared with autopsy are described.

\section{MATERIALS AND METHODS}

The life partner of a 59-year-old man called the paramedics after she found him lying on his bed with blood on his belly. The man was already dead when the paramedics arrived. The decedent had a gunshot wound to the belly and a revolver lying next to him. Immediately, the police, a forensic pathologist, and a prosecutor were summoned. Although the manner of death was deemed suicide because the decedent left a suicide message on the answering machine, the prosecutor commissioned an autopsy and radiologic examination before the autopsy.

The decedent underwent a radiologic examination of the entire body using a 128-slice CT system (SOMATOM Definition Flash; Siemens Healthcare, Forchheim, Germany). The thorax and abdomen were scanned at $120 \mathrm{kVp}$ with dose modulation and a reference value of $400 \mathrm{mAs}$. The raw data were reconstructed with a slice thickness of $1 \mathrm{~mm}$ and an increment of $0.8 \mathrm{~mm}$ using a hard kernel (B60) and a soft kernel (B30). An additional high-resolution scan $(120 \mathrm{kVp}$ and $330 \mathrm{mAs})$ was performed and reconstructed in a small field of view with a slice thickness of $0.4 \mathrm{~mm}$ using a hard kernel (U70) and a soft kernel (U30). ${ }^{8}$

Furthermore, an MRI examination of the abdomen was performed on a 3 Tesla MRI scanner (Achieva 3.0 TX; Philips Medical System, Best, the Netherlands). The MRI protocol consisted of T2-weighted sequences performed in the transverse and sagittal orientation, a T2-weighted spectral attenuated inversion recovery sequence (with fat suppression) in the transverse orientation, a T1-weighted sequence in the transverse orientation, and a $\mathrm{T} 2 *$-weighted sequence (with susceptibility weighting) in the transverse orientation. The slice thickness for all sequences was $3 \mathrm{~mm}$. Finally, the CT and MRI data sets were fused for detailed visualization of the soft tissue injuries as well as the osseous injuries. A board-certified radiologist reviewed the CT and MRI data.

Subsequent to the radiologic examinations, an autopsy was performed. Dissection of the abdomen was performed layer-bylayer according to the bullet path. Then, complete dissection of the thoracic and abdominal organs and the brain was performed. 

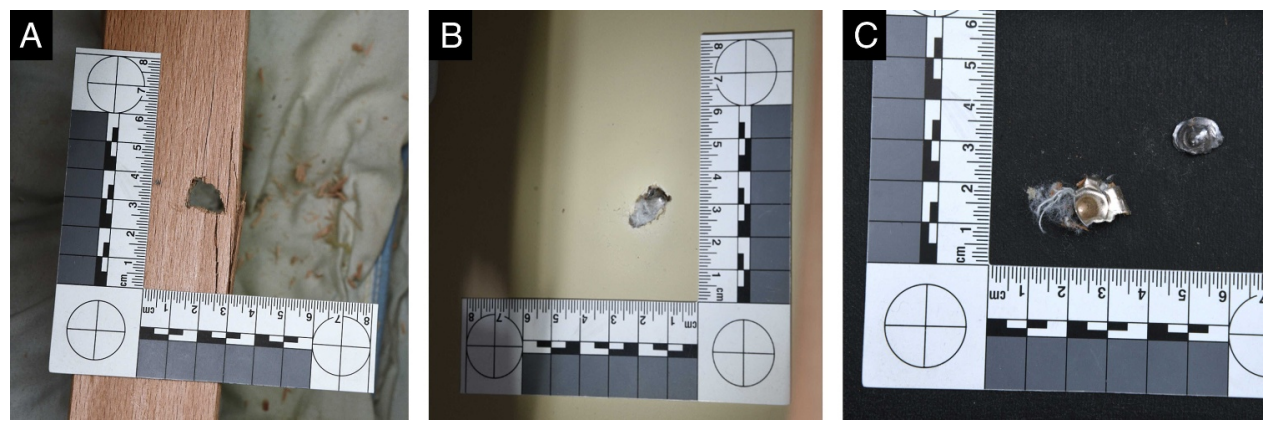

FIGURE 1. The decedent had a gunshot wound to the belly (entrance wound) and a wound on the left side of his back (exit wound). After the projectile exited the body, it penetrated the mattress and an element of the wooden slat frame (A) until it became stuck in a wooden drawer (B) beneath the bed. A deformed and fragmented .357 Magnum bullet was located (C).

A board-certified forensic pathologist and a resident in forensic pathology performed the autopsy.

\section{RESULTS}

On external examination, it was estimated that the time since death was approximately 4 hours. The decedent was not wearing any clothes except his pants. A contact gunshot wound (diameter: $15 \mathrm{~mm} / 0.59 \mathrm{in}$. was detected in almost the center of the epigastric region. Analysis for gunshot residue at the entrance wound using the rhodizonate staining technique yielded a very weakly positive result. The exit wound (diameter: $12 \mathrm{~mm} / 0.47$ in. was located on the left side of the back at a distance of approximately $7 \mathrm{~cm} /$ 2.75 in. from the thoracolumbar transition. After the projectile exited the body, it perforated the mattress and an element of the wooden slat frame until it became lodged in a wooden drawer (Fig. 1). A .357 Magnum revolver (Sturm, Ruger \& Co, Inc) was lying next to his right shoulder. His right arm was angled such that the right hand was lying on the shoulder. Five chambers of the revolver's cylinder were loaded with .357 Magnum (GECO) cartridges (RUAG Ammotec AG, Thun, Switzerland). The remaining sixth chamber held the cartridge case of a .357 Magnum (GECO). The deformed and fragmented projectile obtained from the wooden drawer was finally identified as a full metal jacket .357
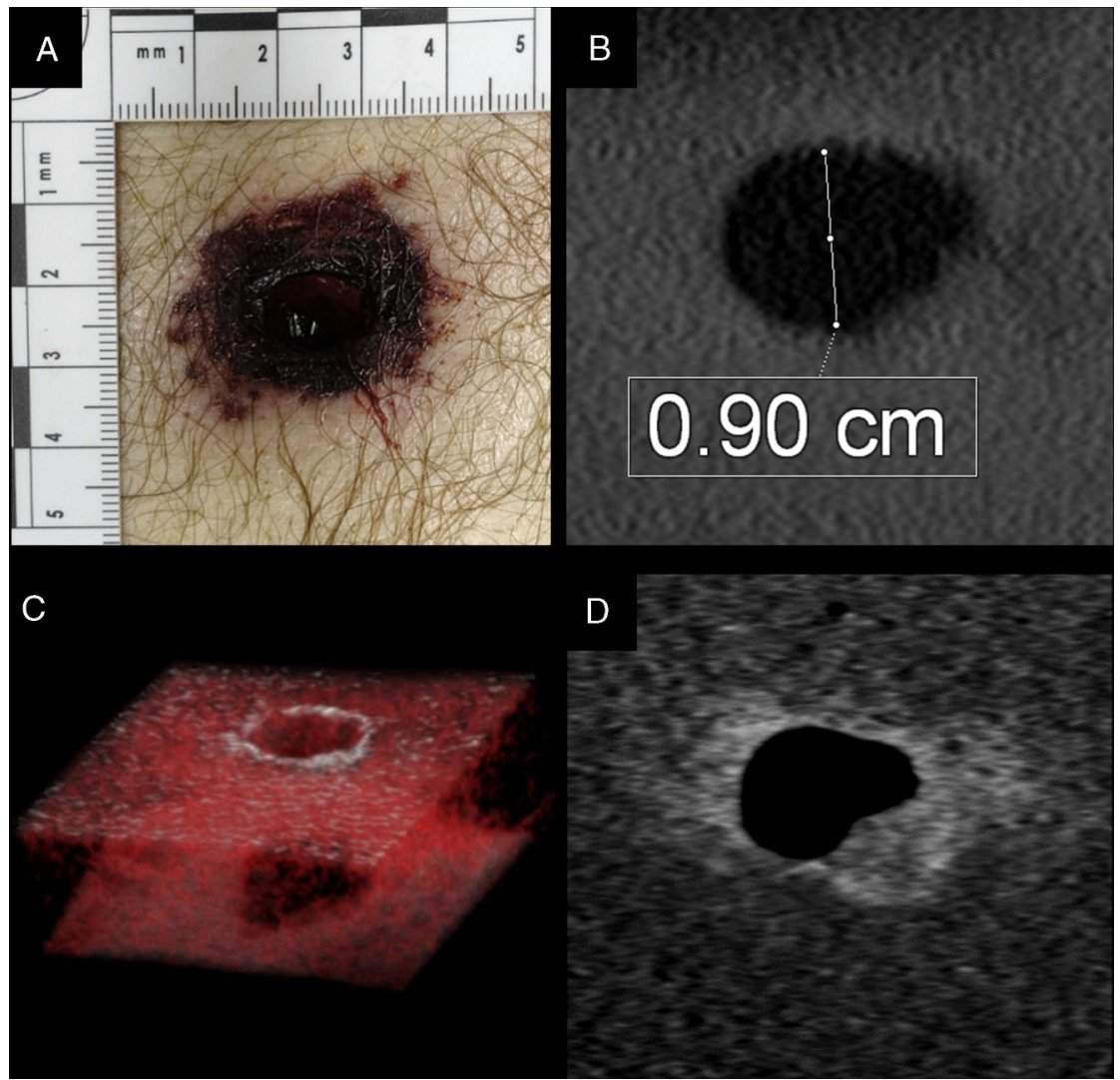

FIGURE 2. The entrance wound demonstrated a muzzle imprint mark (A) consistent with a contact shot. The diameter of the entrance wound was approximately $9 \mathrm{~mm} / 0.35 \mathrm{in}$. on CT (A: coronal view), which was consistent with the caliber size of the secured .357 Magnum bullet. A hyperdense ring could be visualized on high-resolution $\mathrm{CT}$ (C: volume rendering), which might indicate the rim of the muzzle imprint mark. Combustion residue, soot deposition, tissue damage, and blood probably caused the hyperdense region beneath the skin surrounding the entrance hole (D: coronal view). 


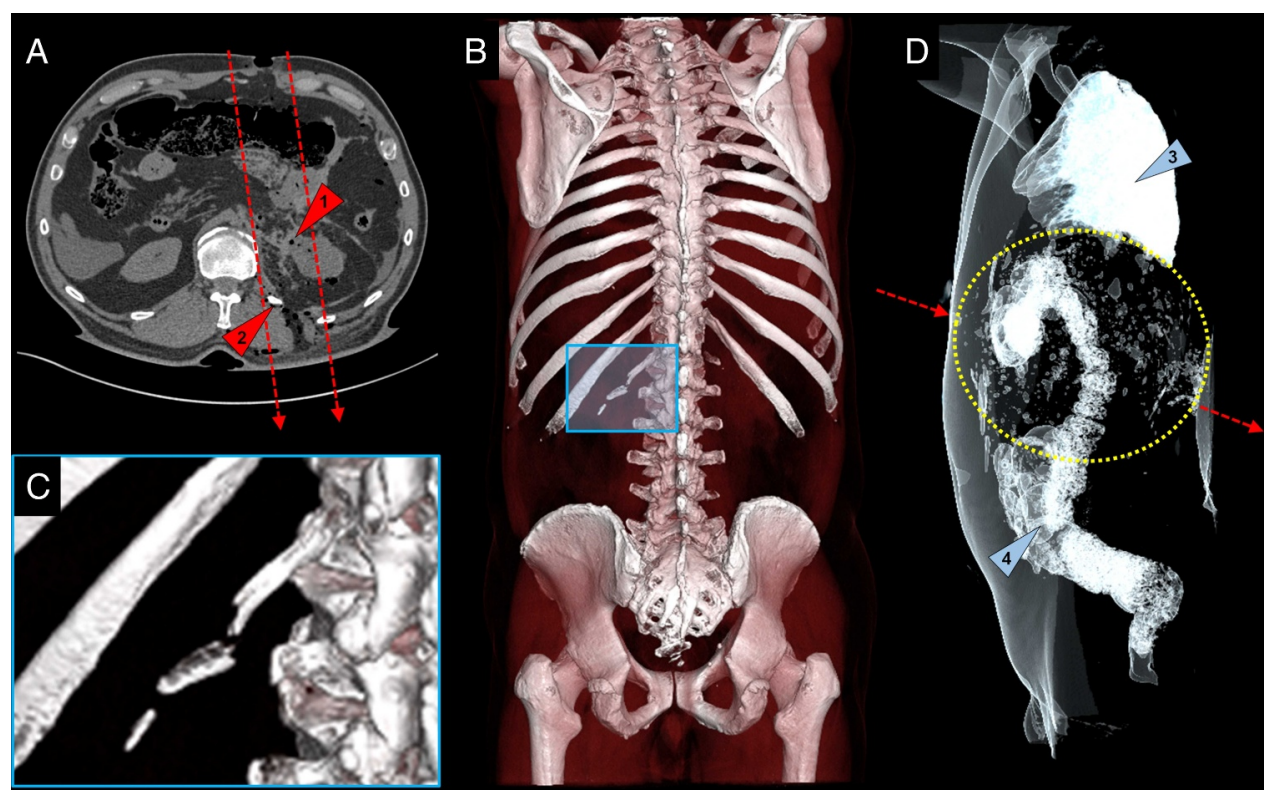

FIGURE 3. The bullet path was indicated on CT (A [red arrows]: axial view) by injury to muscles, the peritoneal fat, and the left kidney (B [arrowhead 1]: axial view) as well as a fracture of the 13th rib (A [arrowhead 2]: axial view/B and C [blue frames]: volume renderings). Small gas cavities were detected ( $D$ [yellow circle]: volume rendering [arrowhead 3: lung, arrowhead 4: colon]), which might be suggestive of the extent of the temporary wound cavity. Computed tomography revealed no remaining bullet fragments in the body.

Magnum bullet. Gunshot residue was not detected on the hands of the decedent or on the hands of the decedent's life partner using the rhodizonate staining technique.

The CT examination was performed 17 hours after the external examination. Defects on the skin and directly beneath the skin were indicated at the entrance (Fig. 2). Defects in the musculus rectus abdomini were detected where the bullet entered the abdomen. Defects in the musculus erector spinae were detected where the bullet exited the body. The bullet trajectory was indicated by defects in the peritoneal fat. An injury to the left kidney was also indicated on CT by small gas cavities in the renal tissue and deformation of the renal tissue. Computed tomography revealed that the decedent had 25 vertebrae and 13 pairs of ribs. A fracture of the 13th rib on the left side was detected on CT (Fig. 3). Numerous small gas cavities were observed spread along and beyond the wound channel (Fig. 3D). Furthermore, hyperdense fluid accumulations were detected on $\mathrm{CT}$, which were located between the intestinal loops as well as dorsal to the liver, spleen, and transverse colon and in the pelvic region. The CT examination excluded the presence of any (potentially ferromagnetic) bullet fragments inside the body, and thus, an MRI examination was approved.
The MRI examination was performed 17 and a half hours after the external examination. The hyperdense fluid accumulations on CT were confirmed as sedimented blood by MRI (Fig. 4). The defects in the muscle tissue and the peritoneal fat as well as the injury to the kidney were clearly identifiable on MRI (Fig. 5). Injuries to organs other than the kidney could not be clearly identified on MRI.

The autopsy was performed 20 hours after the initial external examination. The autopsy confirmed the defects in the muscles where the bullet entered and exited the body, the injury to the left kidney, and the fracture of the 13th rib. In addition, small tears in the liver and spleen were reported during the autopsy. However, the autopsy also revealed that the duodenum was perforated, which was not detected on CT or MRI, although blood was located between the intestinal loops. Furthermore, the autopsy revealed injuries to the left adrenal gland, which were also not clearly identifiable on the radiologic images. At this time, approximately $700 \mathrm{~mL}$ of blood was in the abdominal cavity, and livor mortis has sparsely developed. The right kidney showed dark red medullary pyramids and light red cortical tissue (shock kidney), and subendocardial hemorrhages were detected. These

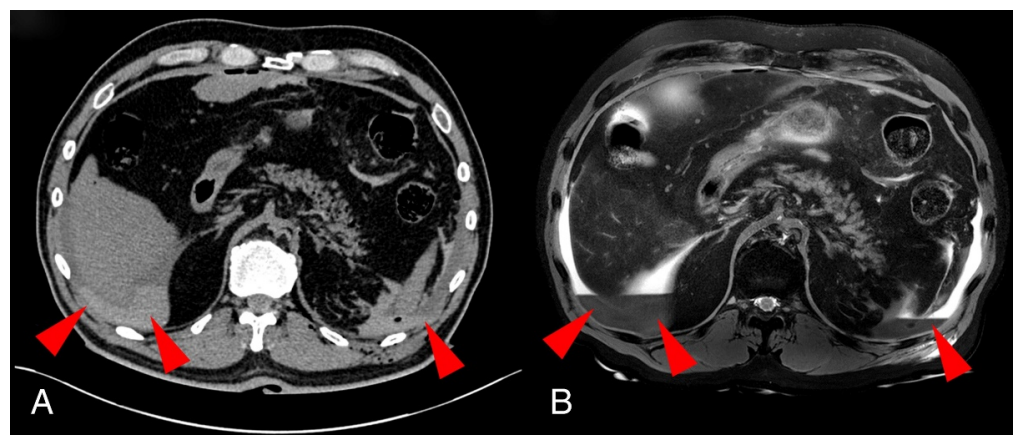

FIGURE 4. A large amount of blood ( $A$ and $B$, red arrowheads: sedimented blood) was located in the abdominal cavity on CT (A: axial view) and on MRI (B: axial view). The amount of blood was more easily detectable on MRI (B: T2-weighted spectral attenuated inversion recovery). 


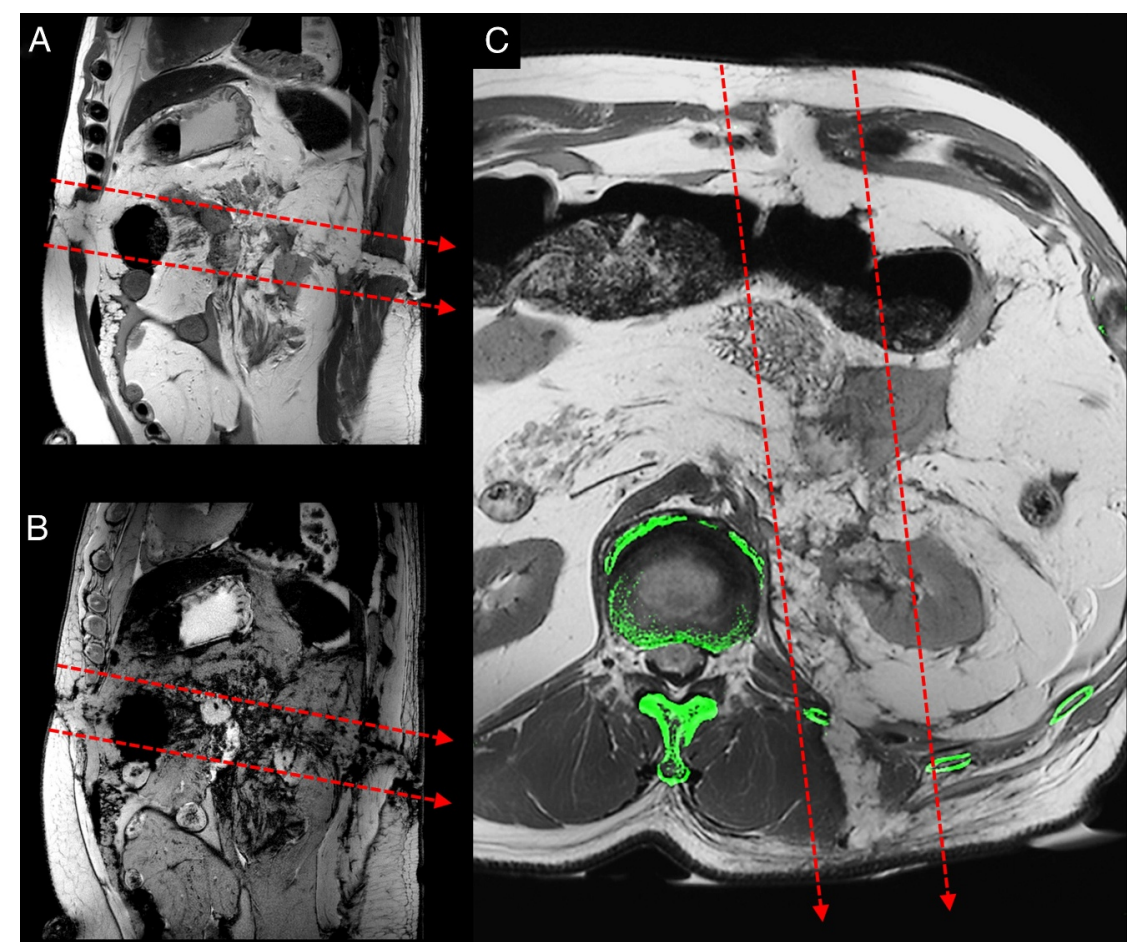

FIGURE 5. Magnetic resonance imaging allowed for a detailed depiction of the soft tissue injuries along the wound channel (A [red arrows]: sagittal view, T2-weighted sequence/B [red arrows]: sagittal view, T2*-weighted sequence). Fusion of the CT dataset (C: axial view, bones are highlighted in green) and the MRI dataset (C: axial view, T2-weighted sequence) allowed for clear visualization of the bullet path (C [red arrows]: axial view).

findings were deemed consistent with exsanguination resulting from a gunshot wound to the abdomen, which was defined as the cause of death.

\section{DISCUSSION}

This case report presents the forensic use of postmortem CT and MRI in a case of an abdominal gunshot wound. The radiologic examinations together with the external examination allowed for identification of the intracorporeal trajectory. The autopsy confirmed the CT and MRI findings. However, the autopsy also revealed findings along the wound tract that had been missed on imaging and presented additional signs of death by exsanguination.

In addition to the exclusion of lodged bullet fragments, postmortem CT may help assess the shot distance, reveal the intracorporeal deflection of a bullet, and indicate the extent of the temporary cavity. The hyperdense ring on the surface and the hyperdense region beneath the skin were indicative of contact-range discharge. These findings were probably caused by the gases coming from the barrel of the firearm and the tissue damage caused by the bullet itself. The presence of combustion residue at the bullet's entry site indicates a contact shot. ${ }^{9}$ The fracture of the 13th rib observed on CT may have been related to slight deflection of the bullet. The small gas cavities might have been caused by the pressure wave during the creation of the temporary cavity. These gas cavities were attributed to small injuries rather than exclusively to decomposition, not only because the postmortem interval was rather short at the time of imaging but also because the gas cavities were solely located in the region surrounding the bullet path. However, decomposition may have supported the development of gas within destroyed tissue. In addition, small tears in the liver and spleen were observed on autopsy. Although soft tissue injuries were already suggested by the CT findings, MRI was beneficial to delineate the wound tract in the subcutaneous soft tissue. The defects in the muscle tissue and peritoneal fat as well as the injury to the left kidney were clearly visible on MRI. Postmortem MRI additionally allowed for a more accurate estimation of the blood volume by depicting the sedimented blood. However, the gas in the bowels, the small gas cavities, and the amount of blood certainly limited the diagnostic value of MRI. The perforated duodenum and the injured adrenal gland detected on autopsy were missed on imaging. Furthermore, the autopsy provided additional findings consistent with exsanguination that were not detected on imaging. The condition of the right kidney and the subendocardial hemorrhages were additional signs of exsanguination, ${ }^{10,11}$ aside from the sparsely developed livor mortis and the large amount of blood in the abdomen cavity.

\section{CONCLUSIONS}

Performing postmortem CT is recommended in cases of gunshot wounds to aid autopsy and to document the findings in situ. Postmortem MRI can be a valuable supplement to postmortem CT concerning the precise delineation of the wound tract and the visualization of soft tissue injuries. Further research on postmortem MRI is desirable to reveal further potential advantages of this imaging technique over CT and autopsy. In certain cases of gunshot injuries, radiologic examinations may be sufficient to detect the bullet trajectory and internal injuries. However, an autopsy cannot be waived for a thorough examination of gunshot injuries. 


\section{REFERENCES}

1. Giorgetti A, Giraudo C, Viero A, et al. Radiological investigation of gunshot wounds: a systematic review of published evidence. Int J Leg Med. 2019;133:1149-1158.

2. van Kan RAT, Haest IIH, Lahaye MJ, et al. The diagnostic value of forensic imaging in fatal gunshot incidents: a review of literature. J Forensic Radiol Imaging. 2017;10:9-14.

3. Thali MJ, Yen K, Vock P, et al. Image-guided virtual autopsy findings of gunshot victims performed with multi-slice computed tomography (MSCT) and magnetic resonance imaging (MRI) and subsequent correlation between radiology and autopsy findings. Forensic Sci Int. 2003; 138:8-16.

4. Andenmatten MA, Thali MJ, Kneubuehl BP, et al. Gunshot injuries detected by post-mortem multislice computed tomography (MSCT): a feasibility study. Leg Med (Tokyo). 2008;10:287-292.

5. Viel G, Gehl A, Sperhake JP. Intersecting fractures of the skull and gunshot wounds. Case report and literature review. Forensic Sci Med Pathol. 2009; $5: 22-27$.
6. Oehmichen M, Gehl H-B, Meissner C, et al. Forensic pathological aspects of postmortem imaging of gunshot injury to the head: documentation and biometric data. Acta Neuropathol. 2003;105:570-580.

7. Gascho D, Tappero C, Zoelch N, et al. Synergy of CT and MRI in detecting trajectories of lodged bullets in decedents and potential hazards concerning the heating and movement of bullets during MRI. Forensic Sci Med Pathol. 2020;16:20-31. https://doi.org/10.1007/s12024-019-00199-y, 1, 12.

8. Gascho D, Thali MJ, Niemann T. Post-mortem computed tomography: technical principles and recommended parameter settings for high-resolution imaging. Med Sci Law. 2018;58:70-82.

9. Schyma C, Bauer K, Brünig J, et al. Visualization of the powder pocket and its influence on staining in firearm barrels in experimental contact shots. Int J Leg Med. 2017;131:167-172.

10. Adebahr G, Weiler G. Distribution of blood in the kidney by death from exsanguination (author's transl) [in German]. Z Rechtsmed. 1977;80:9-16.

11. Plattner T, Yen K, Zollinger U. The value of subendocardial haemorrhages as an indicator of exsanguination and brain injury - a retrospective forensic autopsy study. J Forensic Leg Med. 2008;15:325-328. 